\title{
A!
}

This is an electronic reprint of the original article.

This reprint may differ from the original in pagination and typographic detail.

Maurya, Somendu; Nyman, Markus; Kaivola, Matti; Shevchenko, Andriy

\section{Near-field spatial coherence of structured incoherent optical sources}

Published in:

Physical Review A

DOI:

10.1103/PhysRevA.102.053509

Published: 10/11/2020

Document Version

Publisher's PDF, also known as Version of record

Please cite the original version:

Maurya, S., Nyman, M., Kaivola, M., \& Shevchenko, A. (2020). Near-field spatial coherence of structured

incoherent optical sources. Physical Review A, 102(5), [053509]. https://doi.org/10.1103/PhysRevA.102.053509

This material is protected by copyright and other intellectual property rights, and duplication or sale of all or part of any of the repository collections is not permitted, except that material may be duplicated by you for your research use or educational purposes in electronic or print form. You must obtain permission for any other use. Electronic or print copies may not be offered, whether for sale or otherwise to anyone who is not an authorised user. 


\title{
Near-field spatial coherence of structured incoherent optical sources
}

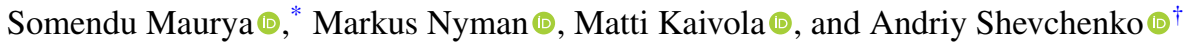 \\ Department of Applied Physics, Aalto University, P.O.Box 13500, FI-00076 Aalto, Finland
}

(Received 18 August 2020; accepted 23 October 2020; published 10 November 2020)

\begin{abstract}
Spatial coherence is a fundamental property of light with many applications. However, if a light source is spatially incoherent and structured, its near-field coherence is difficult to evaluate directly because of the complexity of the emitted random light. In this work, we propose a general approach to calculate the nearfield mutual intensity and transverse coherence length of propagating radiation from a periodically structured incoherent source using optical reciprocity. If the structure period is subwavelength and much smaller than the source, the mutual intensity reduces to a Fourier transform of the angular intensity spectrum of the radiation. Tailoring the near-field spatial coherence of incoherent sources by structuring can be used, e.g., for applications in optical sensing and imaging.
\end{abstract}

DOI: 10.1103/PhysRevA.102.053509

\section{INTRODUCTION}

Characterization of optical radiation sources in terms of their temporal and spatial coherence plays an important role for many applications [1,2]. These applications include optical imaging, sensing and detection, holography, phase sensitive microscopy, and other techniques to study, analyze, and use partially coherent light [3-8]. As an example, spatial coherence lies in the core of many correlation-based imaging techniques, such as full-field optical coherence tomography $[4,9]$, aberration-insensitive interferometric microscopy $[10,11]$, and ghost imaging [12,13]. Moreover, optical beams with designed spatial coherence have been shown to exhibit unusual propagation characteristics, such as self-healing and shape preservation in turbulent atmosphere $[14,15]$.

While there are already well established theoretical foundations to characterize spatial coherence of light in the far-field zone of the source [16], the near-field characterization methods are still quite limited. Recently, some methods have been proposed to characterize theoretically the nearfield coherence of optical sources by taking into account the evanescent and propagating parts of the radiation [17-19]. However, these methods require prior knowledge of the radiation properties of the source, which can be difficult to obtain for nontrivial three-dimensional and structured sources.

If the light source is a distribution of independent pointlike sources, such as fluorescent molecules and quantum dots, its radiation is highly spatially incoherent in the vicinity of the source surface. Since the dipole near fields of the molecules have a very short decay length, on the order of a tenth part of the wavelength, they are of limited practical interest. However, it has been shown that at larger distances, the near-field coherence length of a flat spatially incoherent but uniform source is equal to about $\lambda / 4$ as long as the distance from the

\footnotetext{
*somendu.maurya@aalto.fi

†andriy.shevchenko@aalto.fi
}

source's surface is small compared to the transverse size of the source [20]. If the near field of such a source is used to illuminate an object in an interferometric imaging setup, the resolution will be limited to $\lambda / 4$. However, if the source is structured (e.g., to enhance the directivity of radiation [21]), the near-field coherence length can be different. In general, the possibility to characterize and design the near-field coherence properties of nanostructured optical sources can be of high practical interest.

In this paper, we introduce a semi-analytical model for characterization of near-field coherence properties of microor nanostructured spatially incoherent light sources. The model enables effective estimation of the transverse coherence function and coherence length of an arbitrary periodically structured source and offers the possibility to tailor the structure to obtain the desired coherence properties. The calculation method is based on the optical reciprocity theorem [21-25] that allows us to study the radiation properties and spatial coherence of the source in terms of coupling of external plane waves to the structure. We describe the method in detail in the next section and then demonstrate its application by calculating the coherence length for radiation from several light sources.

\section{THE METHOD}

Spatial coherence of light is determined by the mutual correlation function $G(\mathbf{d})$ of the light field $E(\mathbf{r})$ simultaneously at two points separated by a vector $\mathbf{d}$. This function is also known as the mutual intensity. Here we use a scalar approximation for the field, and later, when we deal with numerical calculations, we will fit the calculated vector fields to the scalar-wave theory. A normalized form of this function is defined as

$$
g(\mathbf{d})=\frac{G(\mathbf{d})}{\sqrt{I(\mathbf{r})} \sqrt{I(\mathbf{r}+\mathbf{d})}}=\frac{\left\langle E^{*}(\mathbf{r}) E(\mathbf{r}+\mathbf{d})\right\rangle}{\sqrt{I(\mathbf{r})} \sqrt{I(\mathbf{r}+\mathbf{d})}},
$$


(a)

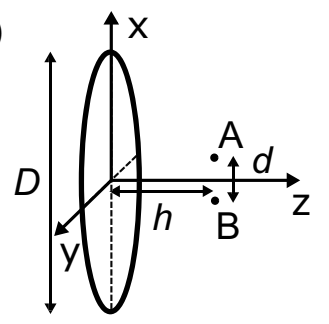

(c)

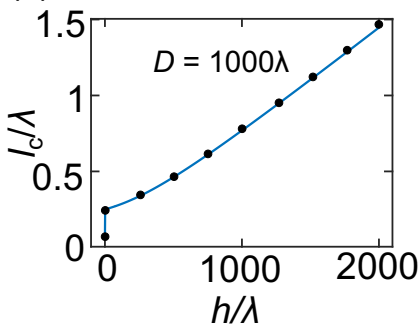

(d)

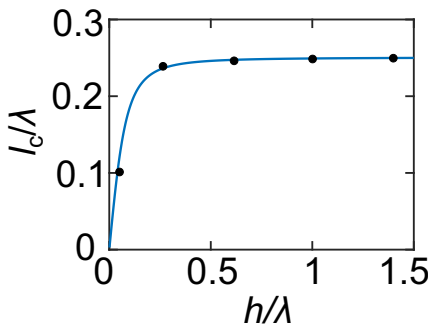

FIG. 1. Spatial coherence of radiation of a spatially incoherent optical source: (a) Schematic of a planar circular source of diameter $D$. Points A and B are in a plane parallel to the source plane at a distance $h$ from the source; (b) The magnitude of the normalized mutual intensity between points A and B separated in the transverse plane by distance $d$ when $h=\lambda$ ( $l_{\mathrm{c}}$ is the coherence length); (c) The transverse coherence length as a function of $h$ for the source in (a) with $D=1000 \lambda$; (d) The dependence of $l_{\mathrm{c}}$ on $h$ in the near field of the source. In (c) and (d), the results of numerical calculations are presented by the black dots fitted by a smooth function (blue line).

where the angle brackets denote time or ensemble averaging and $I(\mathbf{r})$ and $I(\mathbf{r}+\mathbf{d})$ are average intensities at $\mathbf{r}$ and $\mathbf{r}+\mathbf{d}$ [26]. The transverse coherence length is defined as the distance between two points in the transverse plane at which the function $g(\mathbf{d})$ decreases in its absolute value from 1 to $1 / 2$.

Let us consider a spatially incoherent planar light source of a circular shape that has a diameter $D$, as shown in Fig. 1(a). We assume that it consists of uniformly distributed independent point dipoles. For a certain distance $h$ away from the central part of the source, one can evaluate the fields at two points A and B and calculate $g(d)$ as a function of the distance $d$ between these points using Eq. (1). Because Eq. (1) is written for scalar fields, the fields of the dipoles must also be written as scalar functions. We formally assume that this scalar dipole field is equal to the transverse component of the electric field vector of dipole radiation [27], that is

$$
E_{\mathrm{d}}\left(r_{1 \mathrm{~A}}\right)=U\left(\mathbf{r}_{1}\right) \frac{e^{-i k r_{1 \mathrm{~A}}+i \omega t}}{r_{1 \mathrm{~A}}}\left(1-\frac{i}{k r_{1 \mathrm{~A}}}-\frac{1}{k^{2} r_{1 \mathrm{~A}}^{2}}\right),
$$

where $\mathbf{r}_{1}$ is the coordinate of the dipole, $r_{1 \mathrm{~A}}$ is the distance from the dipole to the observation point $\mathrm{A}$, and $U$ is an amplitude of the field that depends on the dipole moment and its orientation. The second and third terms are the two near-field terms of dipole radiation. We note that the dipole near fields also contain the radial component of the electric field, but it

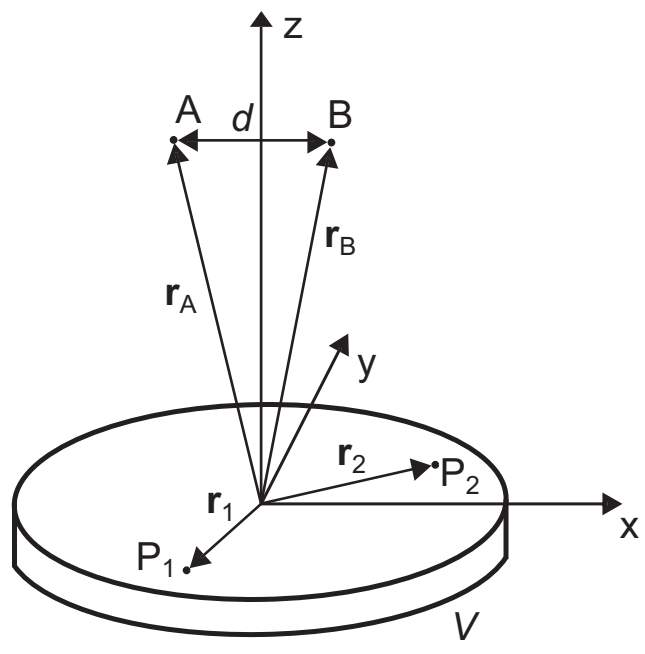

FIG. 2. Schematic of a disk-shaped nanostructured source of volume $V$ consisting of homogeneously distributed statistically independent point emitters. The internal structure of the source is not shown. The coordinate system used is centered at the central point of the source's surface.

has the same form as the second and the third term in Eq. (2) and is significant only at very short distances from the dipole. In the considered source, the dipoles are identical, distributed uniformly, and oriented randomly. Therefore the fluctuating amplitude $U$ is on average the same for all the dipoles. The unnormalized mutual correlation function is then calculated by integrating the contributions of the dipoles to the fields at points $\mathrm{A}$ and $\mathrm{B}$ and inserting these fields into Eq. (1). This procedure leads to the following expression:

$$
\left\langle E_{\mathrm{A}}^{*} E_{\mathrm{B}}\right\rangle=\int_{0}^{2 \pi} \int_{0}^{D / 2}\left\langle E_{\mathrm{d}}^{*}\left(r_{1 \mathrm{~A}}\right) E_{\mathrm{d}}\left(r_{1 \mathrm{~B}}\right)\right\rangle r_{1} d r_{1} d \phi,
$$

where the integration over the source surface is done in polar coordinates $\left(r_{1}, \phi\right)$ with the origin in the center of the source (see also Fig. 2); $r_{1 \mathrm{~A} / \mathrm{B}}$ is the distance between the coordinate $\mathbf{r}_{1}$ and the point A/B. To obtain Eq. (3), we have used the fact that the dipoles are statistically independent and replaced $\left\langle U^{*}\left(\mathbf{r}_{1}\right) U\left(\mathbf{r}_{2}\right)\right\rangle$ with $\left\langle U^{*}\left(\mathbf{r}_{1}\right) U\left(\mathbf{r}_{1}\right)\right\rangle \delta\left(\mathbf{r}_{2}-\mathbf{r}_{1}\right)$, where $\delta\left(\mathbf{r}_{2}-\mathbf{r}_{1}\right)$ is the Dirac delta function. Figure 1(b) shows the normalized mutual correlation function calculated in this way for $z=\lambda$. The coherence length is in this case equal to $0.25 \lambda$. We calculated the coherence length for a number of discrete values of $z$ between 0 and 2000 $\lambda$. The results are shown in Figs. 1(c) and 1(d) by black dots. We noticed that the third term in Eq. (2) influences the calculation results only if the distance $z$ is small compared to $0.1 \lambda$. Hence, if these short distances are not of interest, the third term in Eq. (2) can be neglected. In Figs. 1(c) and 1(d), both near-field terms are taken into account. The blue line in Figs. 1(c) and 1(d) shows fitting of our numerical results with a smooth function. We have found that this function is inversely proportional to the average (root-mean-square) transverse amplitude of the total field evaluated on the z-axis. Although we do not have rigorous derivation of this dependence, we notice that the transverse coherence length at a fixed distance from the source 
can be calculated as

$$
\begin{aligned}
l_{\mathrm{c}} & =K \int_{-\infty}^{\infty}\left|g\left(x_{\mathrm{B}}-x_{\mathrm{A}}\right)\right| d x_{\mathrm{B}} \\
& =\frac{K}{\sqrt{I\left(x_{\mathrm{A}}\right)}} \int_{-\infty}^{\infty}\left|\frac{\left\langle E^{*}\left(x_{\mathrm{A}}\right) E\left(x_{\mathrm{B}}\right)\right\rangle}{\sqrt{I\left(x_{\mathrm{B}}\right)}}\right| d x_{\mathrm{B}},
\end{aligned}
$$

where $K$ is a constant, usually taken to be $1 / 2$. If the integral in the second expression is independent of $z$, we obtain $l_{\mathrm{c}}=$ $K_{1} / \sqrt{I\left(x_{\mathrm{A}}\right)}$, where $K_{1}$ is a new constant that can be used as a fitting parameter and $x_{\mathrm{A}}$ can be set to 0 .

As seen from Fig. 1(d), the coherence length depends on the distance from the source. In the range of $h<0.1 \lambda$, the coherence length sharply increases with $h$ from 0 to about $\lambda / 4$, because the evanescent waves of the point emitters vanish. In the range of $h$ from $0.2 \lambda$ to about $0.1 D$, the coherence length stays approximately at the same level of $\lambda / 4$. Then, at even larger values of $h$, it increases with the distance due to the well known phenomenon of gain of spatial coherence by propagation [26]. In the far field of the source $(h \gg D)$, the coherence length is given in accordance with the Van Cittert-Zernike (VCZ) theorem [26,28].

For certain simple spatially incoherent sources, the radiated field and coherence function can be calculated using already developed theoretical methods [28]. However, any practical approach to treat spatial coherence in the near-field range, where the internal structure of the source can play a significant role, has not yet been introduced. We note that the random-field distribution of a spatially incoherent nanostructured source is difficult if not impossible to calculate analytically or even numerically, because the radiation of each of the independent point emitters will be modified by the structured environment. Therefore, in most cases, Eq. (1) will not be possible to use directly. In the following, we derive a set of equations that allows one to calculate the mutual intensity in practice for such structured light sources. The approach is based on optical reciprocity and can be numerically implemented with the help of any Maxwell's equation solver like COMSOL MULTIPHYSICS used by us in this work.

Consider a spatially incoherent light source consisting of mutually uncorrelated point emitters of scalar waves with the same central angular frequency $\omega$ (see Fig. 2). At some point A above the source, the electric field of light can be written as

$$
E_{\mathrm{A}}=\int_{V} d \mathbf{r}_{1} \iint_{-\infty}^{\infty} d k_{x} d k_{y} a\left(k_{x}, k_{y}, \mathbf{r}_{1}\right) \exp \left(i \mathbf{k} \cdot \mathbf{r}_{\mathrm{A}}\right)
$$

Because we want to apply the reciprocity principle to optical plane waves, we have written the field through the fluctuating complex amplitudes $a\left(k_{x}, k_{y}, \mathbf{r}_{1}\right)$ of the plane-wave components of radiation originating from a point emitter at a coordinate $\mathbf{r}_{1}$ at the surface of the source $(z=0) ; d k_{x y}$ replaces $d k_{x} d k_{y}$, where $k_{x}$ and $k_{y}$ are the $x$ and $y$ components of the wave vector.

The mutual correlation of the electric fields at two points $\mathrm{A}$ and B above the source (see Fig. 2) is described by the mutual intensity

$$
\begin{aligned}
G_{\mathrm{AB}}= & \left\langle E_{\mathrm{A}}^{*} E_{\mathrm{B}}\right\rangle=\int_{V} \int_{V} d \mathbf{r}_{1} d \mathbf{r}_{2} \iint_{-\infty}^{\infty} d k_{x y} \\
& \times \iint_{-\infty}^{\infty} d k_{x y}^{\prime}\left\langle a^{*}\left(k_{x}, k_{y}, \mathbf{r}_{1}\right) a\left(k_{x}^{\prime}, k_{y}^{\prime}, \mathbf{r}_{2}\right)\right\rangle \\
& \times \exp \left(-i \mathbf{k}^{*} \cdot \mathbf{r}_{\mathrm{A}}\right) \exp \left(i \mathbf{k}^{\prime} \cdot \mathbf{r}_{\mathrm{B}}\right)
\end{aligned}
$$

Here, $\mathbf{r}_{2}$ and the primed quantities are associated with the field calculation at point $\mathrm{B}$ and $\mathbf{k}^{*}$ is the complex conjugate of $\mathbf{k}$ whose $z$ component can be complex, as in the case of evanescent waves. The averaged amplitude product $\left\langle a^{*}\left(k_{x}, k_{y}, \mathbf{r}_{1}\right) a\left(k_{x}^{\prime}, k_{y}^{\prime}, \mathbf{r}_{2}\right)\right\rangle$ can be called the mutual angular correlation function in the source [28]. Since the point emitters do not correlate, we can write

$$
\begin{aligned}
& \left\langle a^{*}\left(k_{x}, k_{y}, \mathbf{r}_{1}\right) a\left(k_{x}^{\prime}, k_{y}^{\prime}, \mathbf{r}_{2}\right)\right\rangle \\
& \quad=\delta\left(\mathbf{r}_{2}-\mathbf{r}_{1}\right) a^{*}\left(k_{x}, k_{y}, \mathbf{r}_{1}\right) a\left(k_{x}^{\prime}, k_{y}^{\prime}, \mathbf{r}_{1}\right),
\end{aligned}
$$

where $\delta\left(\mathbf{r}_{2}-\mathbf{r}_{1}\right)$ is the Dirac delta function. Using the integration property of the delta function, we obtain

$$
\begin{aligned}
G_{\mathrm{AB}}= & \int_{V} d \mathbf{r}_{1} \iint_{-\infty}^{\infty} d k_{x y} \iint_{-\infty}^{\infty} d k_{x y}^{\prime} \exp \left(-i \mathbf{k}^{*} \cdot \mathbf{r}_{\mathrm{A}}\right) \\
& \times \exp \left(i \mathbf{k}^{\prime} \cdot \mathbf{r}_{\mathrm{B}}\right) a^{*}\left(k_{x}, k_{y}, \mathbf{r}_{1}\right) a\left(k_{x}^{\prime}, k_{y}^{\prime}, \mathbf{r}_{1}\right) .
\end{aligned}
$$

This expression takes into account both propagating and evanescent waves. It can be used to calculate the coherence length at any distance $h$ from the source, if we know the complete angular spectrum of all point emitters. As we are interested in the near field of the source, approximately in the range $0.2 \lambda<h<0.1 \mathrm{D}$, we can assume that the transverse size of the source is infinite.

Let us consider a planar source that is periodic with periods $\Lambda_{x}$ and $\Lambda_{y}$ in the $x$ and $y$ directions, respectively. The source is perpendicular to the $z$ direction and has a thickness $t$. Due to the periodicity, the amplitude product in Eq. (8) can be written as

$$
\begin{aligned}
& a^{*}\left(k_{x}, k_{y}, \mathbf{r}_{1}+\vec{\Lambda}_{m n}\right) a\left(k_{x}^{\prime}, k_{y}^{\prime}, \mathbf{r}_{1}+\vec{\Lambda}_{m n}\right) \\
& \quad=a^{*}\left(k_{x}, k_{y}, \mathbf{r}_{1}\right) a\left(k_{x}^{\prime}, k_{y}^{\prime}, \mathbf{r}_{1}\right) \exp \left(i\left(\mathbf{k}^{*}-\mathbf{k}^{\prime}\right) \cdot \vec{\Lambda}_{m n}\right),
\end{aligned}
$$

where $\vec{\Lambda}_{m n}=\left(m \Lambda_{x}, n \Lambda_{y}, 0\right)$ and $m$ and $n$ are integers. The volume integral in Eq. (8), i.e.,

$$
I\left(k_{x}, k_{y}, k_{x}^{\prime}, k_{y}^{\prime}\right)=\int_{V} d \mathbf{r}_{1} a^{*}\left(k_{x}, k_{y}, \mathbf{r}_{1}\right) a\left(k_{x}^{\prime}, k_{y}^{\prime}, \mathbf{r}_{1}\right),
$$

can therefore be written as

$$
\begin{aligned}
I\left(k_{x}, k_{y}, k_{x}^{\prime}, k_{y}^{\prime}\right)= & \sum_{m=-M}^{M} \sum_{n=-N}^{N} \exp \left(i\left(\mathbf{k}^{*}-\mathbf{k}^{\prime}\right) \cdot \vec{\Lambda}_{m n}\right) \\
& \times \int_{v} d \mathbf{r}_{1} a^{*}\left(k_{x}, k_{y}, \mathbf{r}_{1}\right) a\left(k_{x}^{\prime}, k_{y}^{\prime}, \mathbf{r}_{1}\right),
\end{aligned}
$$

where $v$ is the volume of a single unit cell and the source is formally divided into $2 M+1$ and $2 N+1$ unit cells in the $x$ and $y$ directions, respectively. If we assume for simplicity that the structure is periodic only in the $x$ direction, the sum in 
Eq. (11) becomes

$$
\sum_{m=-M}^{M} e^{i\left(k_{x}-k_{x}^{\prime}\right) m \Lambda_{x}}=\frac{\sin \left((M+0.5) \Lambda_{x}\left(k_{x}-k_{x}^{\prime}\right)\right)}{\sin \left(0.5 \Lambda_{x}\left(k_{x}-k_{x}^{\prime}\right)\right)} .
$$

For a large transverse size of the structure, we can set $M \rightarrow$ $\infty$, which allows us to write the above equation in the form

$$
\begin{gathered}
\lim _{M \rightarrow \infty} \frac{\sin \left((M+0.5) \Lambda_{x}\left(k_{x}-k_{x}^{\prime}\right)\right)}{\sin \left(0.5 \Lambda_{x}\left(k_{x}-k_{x}^{\prime}\right)\right)} \\
\quad=C_{1} \sum_{p=-\infty}^{\infty} \delta\left(k_{x}-k_{x}^{\prime}-\frac{2 \pi p}{\Lambda_{x}}\right) .
\end{gathered}
$$

Here, $p$ is an integer associated with the diffraction orders of the structure and $C_{1}$ is a constant that is not relevant, because the function $G_{\mathrm{AB}}$ will eventually be normalized. Now, allowing the structure to be periodic also in the $y$ direction, we obtain

$$
\begin{aligned}
I\left(k_{x}, k_{y}, k_{x}^{\prime}, k_{y}^{\prime}\right)= & C_{1} C_{2} \sum_{p=-\infty}^{\infty} \sum_{q=-\infty}^{\infty} \delta\left(k_{x}-k_{x}^{\prime}-\frac{2 \pi p}{\Lambda_{x}}\right) \\
& \times \delta\left(k_{y}-k_{y}^{\prime}-\frac{2 \pi q}{\Lambda_{y}}\right) \\
& \times \int_{v} d \mathbf{r}_{1} a^{*}\left(k_{x}, k_{y}, \mathbf{r}_{1}\right) a\left(k_{x}^{\prime}, k_{y}^{\prime}, \mathbf{r}_{1}\right),
\end{aligned}
$$

where $C_{2}$ is a coefficient similar to $C_{1}$. Hence, the mutual intensity given by Eq. (8) becomes

$$
\begin{aligned}
G_{\mathrm{AB}}= & C \sum_{p=-\infty}^{\infty} \sum_{q=-\infty}^{\infty} \exp \left(-i \frac{2 \pi p}{\Lambda_{x}} x_{\mathrm{B}}\right) \\
& \times \exp \left(-i \frac{2 \pi q}{\Lambda_{y}} y_{\mathrm{B}}\right) \iint_{-\infty}^{\infty} d k_{x y} \exp \left(i k_{x}\left(x_{\mathrm{B}}-x_{\mathrm{A}}\right)\right) \\
& \times \exp \left(i k_{y}\left(y_{\mathrm{B}}-y_{\mathrm{A}}\right)\right) \exp \left(-i k_{z}^{*} z_{\mathrm{A}}\right) \exp \left(i k_{z}^{(p q)} z_{\mathrm{B}}\right) \\
& \times \int_{v} d \mathbf{r}_{1} a^{*}\left(k_{x}, k_{y}, \mathbf{r}_{1}\right) a\left(k_{x}-\frac{2 \pi p}{\Lambda_{x}}, k_{y}-\frac{2 \pi q}{\Lambda_{y}}, \mathbf{r}_{1}\right) .
\end{aligned}
$$

Here we have $C=C_{1} C_{2}, \mathbf{r}_{\mathrm{A}}=\left(x_{\mathrm{A}}, y_{\mathrm{A}}, z_{\mathrm{A}}\right), \mathbf{r}_{\mathrm{B}}=\left(x_{\mathrm{B}}, y_{\mathrm{B}}, z_{\mathrm{B}}\right)$, and

$$
k_{z}^{(p q)}=\sqrt{k_{0}^{2}-\left(k_{x}-\frac{2 \pi p}{\Lambda_{x}}\right)^{2}-\left(k_{y}-\frac{2 \pi q}{\Lambda_{y}}\right)^{2}} .
$$

Equation (15) takes into account all the diffraction orders of the structure including evanescent waves. To make the analysis more convenient, we can use a modified Weyl identity to write

$$
a\left(k_{x}, k_{y}, \mathbf{r}_{1}\right)=\frac{A\left(k_{x}, k_{y}, \mathbf{r}_{1}\right)}{k_{z}} \exp \left(-i \mathbf{k} \cdot \mathbf{r}_{1}\right),
$$

where $A\left(k_{x}, k_{y}, \mathbf{r}_{1}\right)$ is the complex amplitude function that depends on $k_{x}$ and $k_{y}$, reflecting the fact that the structure can modify the emitted wave profile. Then Eq. (15) can be written as

$$
\begin{aligned}
G_{\mathrm{AB}}= & C \sum_{p=-\infty}^{\infty} \sum_{q=-\infty}^{\infty} \exp \left(-i \frac{2 \pi p}{\Lambda_{x}} x_{\mathrm{B}}\right) \\
& \times \exp \left(-i \frac{2 \pi q}{\Lambda_{y}} y_{\mathrm{B}}\right) \iint_{-\infty}^{\infty} d k_{x y} \exp \left(i k_{x}\left(x_{\mathrm{B}}-x_{\mathrm{A}}\right)\right) \\
& \times \exp \left(i k_{y}\left(y_{\mathrm{B}}-y_{\mathrm{A}}\right)\right) \exp \left(-i k_{z}^{*} z_{\mathrm{A}}\right) \exp \left(i k_{z}^{(p q)} z_{\mathrm{B}}\right) \\
& \times \int_{v} d \mathbf{r}_{1} \frac{A^{*}\left(k_{x}, k_{y}, \mathbf{r}_{1}\right) A\left(k_{x}-\frac{2 \pi p}{\Lambda_{x}}, k_{y}-\frac{2 \pi q}{\Lambda_{y}}, \mathbf{r}_{1}\right)}{k_{z}^{*} k_{z}^{(p q)}} \\
& \times \exp \left(i \frac{2 \pi p x}{\Lambda_{x}}\right) \exp \left(i \frac{2 \pi q y}{\Lambda_{y}}\right) \exp \left(i\left(k_{z}^{*}-k_{z}^{(p q)}\right) z\right),
\end{aligned}
$$

where the emitter coordinate is $\mathbf{r}_{1}=(x, y, z)$. If the order $p$ is larger than $2 \Lambda_{x} / \lambda$ and/or $q$ is larger than $2 \Lambda_{y} / \lambda$, the associated plane wave is evanescent and can be neglected, if the distance from the source surface is large enough. This means that for the structure periods comparable or smaller than the wavelength, only the few lowest orders need to be considered.

For a structured source, the plane-wave amplitudes in the integral of Eq. (18) depend on the propagation direction in a nontrivial manner. In order to be able to calculate them numerically we propose to make use of optical reciprocity. The reciprocity principle requires that, at any point $\mathrm{Q}$, the amplitude of the wave radiated by a point source at a coordinate $\mathrm{P}$ [see Fig. 3(a)] is equal to the amplitude of the wave at point $\mathrm{P}$ that would be radiated by an identical source, if it was located at point $\mathrm{Q}$. The reciprocity is valid also for each plane-wave component of the field at $\mathrm{Q}$, as a consequence of the same two-point reciprocity, but with the second point, Q', located at infinity in the direction of the plane-wave propagation ( $Q^{\prime}$ is shown in Fig. 3(b) schematically) [21,22,25]. Hence, in our numerical calculations, we can send each radiated plane-wave component (with an amplitude $E_{\mathrm{Q}}\left(k_{x}, k_{y}\right)$ and given $k_{x}$ and $k_{y}$ ) back to the source and evaluate the field amplitude $E_{0}\left(\mathbf{r}_{1}\right)$ at each point $\mathrm{P}$ [see Fig. 3(b)]. This amplitude, when normalized to the amplitude of the incident plane wave, will give the contribution of the point source at $\mathrm{P}$ to the considered planewave component at $\mathrm{Q}$. Therefore the obtained normalized amplitudes $E_{0}\left(\mathbf{r}_{1}\right) / E_{\mathrm{Q}}\left(k_{x}, k_{y}\right)$ can be used directly in Eq. (18), replacing the unknown amplitudes $A\left(k_{x}, k_{y}, \mathbf{r}_{1}\right)$ :

$$
A\left(k_{x}, k_{y}, \mathbf{r}_{1}\right)=\frac{E_{0}\left(\mathbf{r}_{1}\right)}{E_{\mathrm{Q}}\left(k_{x}, k_{y}\right)} .
$$

In our numerical calculations we set $E_{\mathrm{Q}}\left(k_{x}, k_{y}\right)$ equal to 1 . The major advantage of this approach is that in the numerical calculations, one can consider a single unit cell with periodic boundary conditions. This is a very light calculation compared to the calculation of the emission pattern of a point source. Figure 3(c) shows a numerically calculated electric field distribution of a wave radiated by a point source at a position $\mathrm{P}$ inside a nanostructure. The wave is seen to be a considerably modified spherical wave. The modification prevents using analytical calculations for evaluation of the mutual intensity $G_{\mathrm{AB}}$. Figure 3(d) illustrates coupling of a plane wave to the structure at an incidence angle of $21^{\circ}$. The amplitude 
(a)
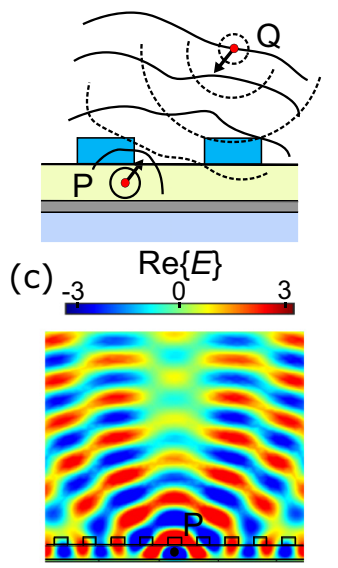

(b)

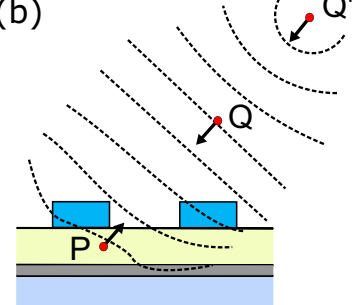

(d)

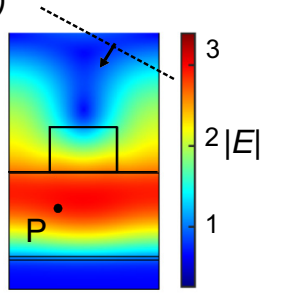

FIG. 3. Explanation of the reciprocity principle. (a) At a point Q, the amplitude of a wave radiated by a point source at a position $\mathrm{P}$ is the same as the amplitude at a point $\mathrm{P}$ of a wave radiated by an identical point source at a position Q. (b) A plane-wave component of light radiated by a point source at $\mathrm{P}$ has at a position $\mathrm{Q}$ the same amplitude as a plane-wave component of light at a position $P$ radiated by a point source at Q. (c) An example of the distribution of the real part of the electric field for a wave radiated by a point dipole located at a position $\mathrm{P}$ inside a nanostructured source made of glass on a reflective metal surface. (d) The electric-field amplitude distribution in the structure of (c) when a plane wave is incident on the structure from above at an angle of $21^{\circ}$. The quantities in (c) and (d) are measured in $\mathrm{V} / \mathrm{m}$.

distribution inside the source gives the relative contribution $A\left(k_{x}, k_{y}, \mathbf{r}_{1}\right)$ of each point source at each point $\mathrm{P}$ to the considered plane wave. Calculating the amplitude distributions for a large number of possible $k_{x}$ and $k_{y}$, we can then evaluate $G_{\mathrm{AB}}$ using Eq. (18).

The developed theory is for scalar fields and numerical calculations using COMSOL MULTIPHYSICS are vector in nature. Therefore, in each step, the TE- and TM-polarized incident waves are considered separately and the functions $\left|A\left(k_{x}, k_{y}, \mathbf{r}_{1}\right)\right|$ are calculated by numerically averaging the obtained two electric-field vectors at each $\mathbf{r}_{1}$ over their all possible orientations. This fits the COMSOL vector-field calculations to our scalar-wave model.

\section{EXAMPLES}

We apply the theory to several examples of light sources. The first example is used to study the influence of a simple periodic patterning of a fluorescent thin film on the near-field intensity profile and the spatial coherence of the emission. We assume that the patterned film consists of thin periodic stripes of width $w$ and period $\Lambda_{x}$. Each point within the stripes contains a point source whose radiation pattern is an undisturbed spherical wave. Because in this example the fields emitted by the point sources are known, neither numerical calculations based on COMSOL MULTIPHYSICS nor the use of optical reciprocity are needed. Figure 4 shows the intensity profile and the normalized mutual intensity for a structure with $w=0.1 \lambda$ and $\Lambda_{x}=3 \lambda$ at two different distances from the source, at $h=$
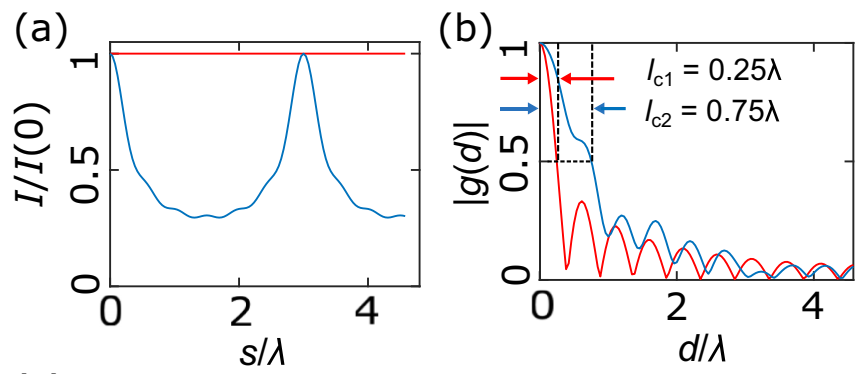

(c)

(d)
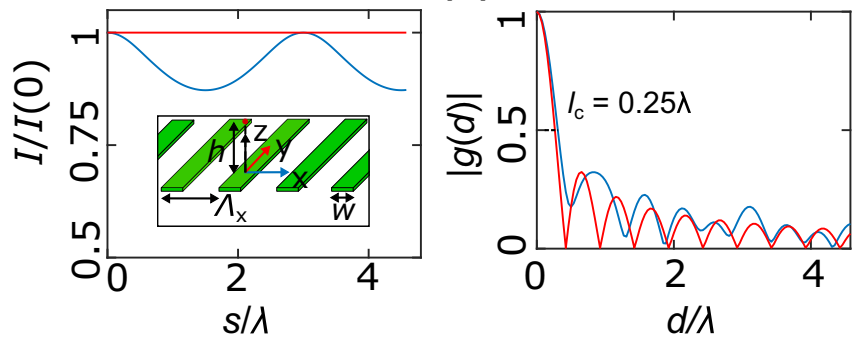

FIG. 4. The intensity profile and spatial coherence of light emitted by a periodically patterned fluorescent thin film [see inset in (c)]. The source consists of narrow stripes of width $w=0.1 \lambda$ and period $\Lambda_{x}=3 \lambda$. Plots (a) and (b) show the intensity and normalized mutual intensity at a distance $h=0.2 \lambda$ and (c) and (d) show the same for $h=1 \lambda$. The inset in (c) shows the orientation of the stripes with respect to the axes of the coordinate system. Blue (light gray)/red (dark gray) curves show the variation of the quantities along the $x / y$ axis. The coordinate $s$ is equivalent to $x$ for blue (light gray) lines and to $y$ for red (dark gray) lines.

$0.2 \lambda$ and $h=1 \lambda$. The blue (light gray) line corresponds to the change of the coordinate along the $x$ axis and the red (dark gray) line along the $y$ axis. The calculations take into account orders $p \in\{-1,0,1\}$. As expected, we observe a periodic intensity variation above the structure along the $x$ direction [see Fig. 4(a) and 4(c)]. This variation, however, diminishes as $h$ increases, because the higher-order plane-wave components become weaker. The calculated normalized mutual intensity shows an obvious anisotropy in the $x y$ plane [see Figs. 4(b) and 4(c)] with the transverse coherence lengths along the $x$ and $y$ axes being respectively equal to $0.25 \lambda$ and $0.75 \lambda$ at $h=0.2 \lambda$. At $h=1 \lambda$, the anisotropy is less pronounced and the coherence length is approximately equal to that of an unpatterned film. It is surprising that the anisotropy essentially disappears already at such a short distance from the film. At this distance, the point sources in both the stripe under the point of measurement and the neighboring stripes contribute significantly to the field in the directions along and perpendicular to the stripes, which is seen also in a considerably flattened intensity profile in Fig. 4(c).

While in the above example, the analytical expression for the field emitted by each point source was known, our further examples require numerical calculations. The functions $A\left(k_{x}, k_{y}, \mathbf{r}_{1}\right)$ in each example have been calculated numerically using COMSOL MULTIPHYSICS by considering coupling of optical plane waves to the structure. The plane-wave incidence angles, which are the polar angle $\theta$ and the azimuthal angle $\phi$, were scanned in small steps from 0 to $\pi / 2$ (also the azimuthal angle was limited by $\pi / 2$ due to the symmetry of 

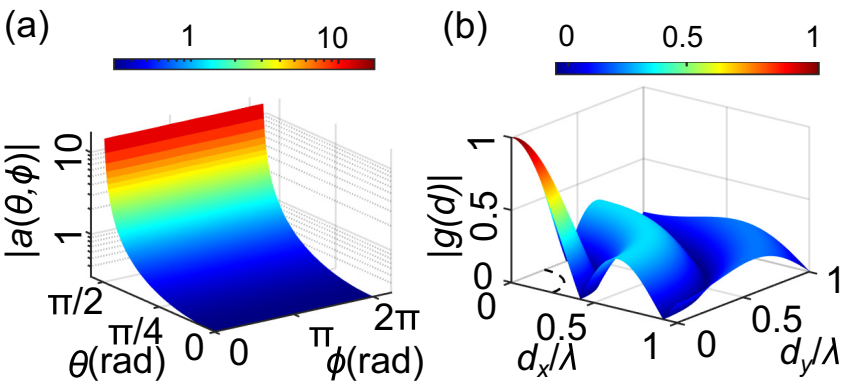

FIG. 5. Two-dimensional plots of (a) the function $|a(\theta, \phi)|$ and (b) the function $|g(d)|$, where $d$ is the length of vector $\mathbf{d}=\mathbf{r}_{\mathrm{A}}-\mathbf{r}_{\mathrm{B}}$. The dashed line shows the distance $d$ at $|g(d)|=0.5$. The function $|a(\theta, \phi)|$ is measured in volts.

the considered structures). Numerical averaging is done over TE- and TM-polarized modes to obtain the effective scalar field amplitudes.

In order to verify the reciprocity-based numerical approach, we first calculate the near-field mutual intensity for a uniform two-dimensional incoherent source shown in Fig. 1(a). Since the point emitters of the source produce independent and identical spherical waves, we need to calculate $\left|a\left(k_{x}, k_{y}, \mathbf{r}_{1}\right)\right|=\left|A\left(k_{x}, k_{y}, \mathbf{r}_{1}\right)\right| / k_{z}$ only at one point. From the Weyl identity we know that $\left|A\left(k_{x}, k_{y}, \mathbf{r}_{1}\right)\right|=\left|A\left(\mathbf{r}_{1}\right)\right|$. Figure 5(a) shows the obtained function $\left|a\left(k_{x}, k_{y}, \mathbf{r}_{1}\right)\right| \equiv$ $|a(\theta, \phi)|$. It increases with $\theta$ and is independent of $\phi$, as expected. Equation (18) then results in the normalized mutual intensity shown in Fig. 5(b). The calculation is seen to reproduce the curve of Fig. 1(b), thereby verifying our semianalytical approach. The dashed line in Fig. 5(b) shows the coherence area corresponding to $l_{\mathrm{c}}=0.25 \lambda$ in Fig. 1(b).

Our next example is a thin-film fluorescent source designed to enhance the directivity of radiation. Figure 6(a) shows the structure of the source. It enhances the fluorescence (including the enhancement in pumping) in the direction normal to the structure by a factor of 350 [21]. The spread angle of the radiation is $\theta_{\mathrm{fl}}=2 \Delta \theta=40^{\circ}$. The fluorescent film, e.g., a dye-doped polymer, has a thickness of $200 \mathrm{~nm}$ in our example. It is sandwiched between two 30- and 50-nm-thick gold layers and placed on a glass substrate. The gold layers act as partially reflecting mirrors forming a Fabry-Perot resonator for the
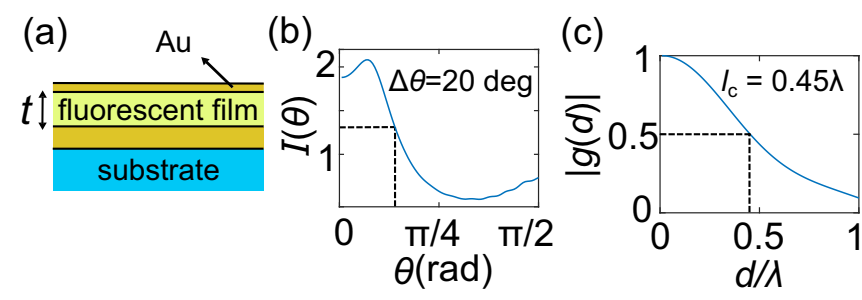

FIG. 6. Spatial coherence of light emitted by a multilayered fluorescent structure. The structure is shown in (a). A fluorescent film of thickness $t=200 \mathrm{~nm}$ is sandwiched between two 30- and 50-nm-thick gold layers on a glass substrate. The calculated function $I(\theta)$ is shown in (b), and (c) illustrates the absolute value of the nearfield coherence function $g(d)$. In (b), the quantity $I(\theta)=|A(\theta)|^{2}$ is measured in $\mathrm{V}^{2} / \mathrm{m}^{2}$.

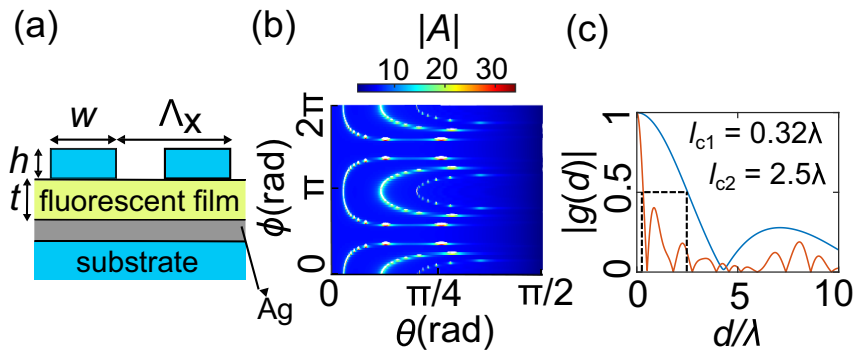

FIG. 7. Spatial coherence of light emitted by a nanostructured fluorescent source. The structure is shown in (a). It consists of a fluorescent film with $t=290 \mathrm{~nm}$ sandwiched between a $50 \mathrm{~nm}$ thick reflecting silver film and a glass grating. The grating parameters are $h=160 \mathrm{~nm}, \Lambda_{x}=480 \mathrm{~nm}$, and $w=220 \mathrm{~nm}$. The calculated $|A|$ is shown in (b), and the red (dark gray) curve in (c) shows the function $|g(d)|$, which gives $l_{\mathrm{c}}=0.32 \lambda$. The blue (light gray) curve shows $|g(d)|$ in the presence of only one diffracted mode, in which case $l_{\mathrm{c}}=2.5 \lambda . \operatorname{In}(\mathrm{b}),|A|$ is measured in $\mathrm{V} / \mathrm{m}$.

emission at a wavelength of $780 \mathrm{~nm}$. A detailed description of the enhancement mechanism can be found in [21]. Figure 6(b) shows the overall angular intensity spectrum $I(\theta)=|A(\theta)|^{2}$ averaged over the fluorescent film. Due to the symmetry of the structure this function is independent of $\phi$. The directivity enhancement is seen in the localization of $I(\theta)$ around $\theta=0$. The mutual intensity is calculated using Eq. (18) and its normalized absolute value is plotted in Fig. 6(c). The coherence length for the structure is equal to $0.45 \lambda$ that is larger than $l_{\mathrm{c}}$ obtained in the previous example (see Fig. 4). Thus, a reduction in the width of the angular intensity spectrum of the source has led to an increase of the near-field transverse coherence length.

The third light source we consider contains a simple periodic surface pattern [see Fig. 7(a)]. A 290-nm-thick fluorescent film $(\lambda=780 \mathrm{~nm})$ is placed on a 50 -nm-thick layer of silver on a glass substrate. A periodic array of glass stripes is then created on the film surface. The thickness, width, and period of the stripes are 160, 220, and $480 \mathrm{~nm}$, respectively. This periodic diffraction grating couples the waveguide modes appearing in the fluorescent film to free-space radiation. The resulting out-coupled waves exhibit narrow angular spreads. The structure supports three waveguide modes with the mode propagation constants of $1.53,1.267$, and 1.04. At $\phi=0$, these modes radiate with the peak intensity observed at $\theta=$ $5^{\circ}, 21^{\circ}$, and $36^{\circ}$. Figure 7 (b) shows the calculated angular amplitude spectrum $|A(\theta, \phi)|$ averaged over a unit cell of the film that exhibits three out-coupled modes at each fixed $\phi$. We note that in addition to these three positive out-coupled modes (with $k_{x}>0$ ), there are also symmetric negative outcoupled modes (with $k_{x}<0$ ). We also note that no pair of the strong out-coupled waves (positive or negative) satisfies the condition $k_{x} \pm k_{x}^{\prime}=2 \pi / \Lambda_{x}$ for phase-matched propagating waves, which means that all the higher orders in $p$ for this structure can be neglected. Our calculation method allows considering the diffracted modes separately. For example, if we consider only one diffracted mode with $k_{x}>0$ (corresponding to $\theta=21^{\circ}$ at $\phi=0^{\circ}$ ), we obtain the blue (light gray) curve in Fig. 7(c) for the function $|g(d)|$, where $d$ is the distance along the $x$ axis. The corresponding transverse 
coherence length is in this case $l_{\mathrm{c}}=2.5 \lambda$ that is much longer than in the previous example due to the enhanced directivity of the radiation. The full width at half maximum of this mode is about $2^{\circ}$. If we take into account also the other diffracted modes, we obtain a rather nontrivial mutual intensity shown by the red (dark grey) curve in Fig. 7(c). The coherence length is now equal to $0.32 \lambda$. This example demonstrates an unexpected short coherence length of a highly directive multibeam radiation from a spatially incoherent source and proves that nanostructuring can be used to manipulate its near-field spatial coherence.

\section{CONCLUSIONS}

In this work, we have developed a method to calculate the normalized mutual intensity of near-field radiation of an incoherent nanostructured planar optical source. The method is based on optical reciprocity and allows one to treat the radiation semianalytically in terms of the plane-wave coupling of light to the source structure. We have shown that, for periodically patterned sources with a large number of unit cells, the near-field mutual intensity can relatively easily be calculated even for complex structures. This opens up a way to control the near-field spatial coherence, e.g., by adjusting the angular spectrum of the emitted light with the help of nanostructures. We have demonstrated a pronounced anisotropy and unexpected lengths of transverse spatial coherence in the near-field zone of structured optical sources. The calculation method can be further developed to take into account also evanescent waves of the point dipolelike sources [as in Eq. (8)], which should allow for obtaining transverse coherence lengths beyond the free-space diffraction limit.

\section{ACKNOWLEDGMENTS}

This work was funded by the Academy of Finland (308394). We also acknowledge the Academy of Finland Flagship Programme, Photonics Research and Innovation (PREIN).
[1] R. Hui and M. O'Sullivan, in Fiber Optic Measurement Techniques, edited by R. Hui and M. O'Sullivan (Academic Press, Boston, 2009), pp. 259-363.

[2] E. Wolf, Coherence and radiometry, J. Opt. Soc. Am. 68, 6 (1978).

[3] Y. L. Li and J. J. Dahl, Angular coherence in ultrasound imaging: Theory and applications, J. Acoust. Soc. Am. 141, 1582 (2017).

[4] Y. Deng and D. Chu, Coherence properties of different light sources and their effect on the image sharpness and speckle of holographic displays, Sci. Rep. 7, 1 (2017).

[5] C. Edwards, B. Bhaduri, T. Nguyen, B. G. Griffin, H. Pham, T. Kim, G. Popescu, and L. L. Goddard, Effects of spatial coherence in diffraction phase microscopy, Opt. Express 22, 5133 (2014).

[6] G. Gbur and O. Korotkova, Angular spectrum representation for the propagation of arbitrary coherent and partially coherent beams through atmospheric turbulence, J. Opt. Soc. Am. A 24, 745 (2007).

[7] C. Balas, Review of biomedical optical imaging - a powerful, non-invasive, non-ionizing technology for improving in vivo diagnosis, Meas. Sci. Technol. 20, 104020 (2009).

[8] D. J. Brady, Optical Imaging and Spectroscopy (Wiley, Hoboken, 2009).

[9] A. Dubois, K. Grieve, G. Moneron, R. Lecaque, L. Vabre, and C. Boccara, Ultrahigh-resolution full-field optical coherence tomography, Appl. Opt. 43, 2874 (2004).

[10] E. Ilina, M. Nyman, I. Švagždyte, N. Chekurov, M. Kaivola, T. Setälä, and A. Shevchenko, Aberration-insensitive microscopy using optical field-correlation imaging, APL Photonics 4, 066102 (2019)

[11] E. Ilina, M. Nyman, T. Mondal, M. Kaivola, T. Setälä, and A. Shevchenko, Interferometric imaging of reflective microobjects in the presence of strong aberrations, Opt. Express 28, 1817 (2020).
[12] D. Simon and A. Sergienko, Correlated-photon imaging with cancellation of object-induced aberration, J. Opt. Soc. Am. B 28, 247 (2011).

[13] Z. Yang, L. Zhao, X. Zhao, W. Qin, and J. Li, Lensless ghost imaging through the strongly scattering medium, Chin. Phys. B 25, 024202 (2015).

[14] O. Korotkova, Random Light Beams: Theory and Applications (CRC, Boca Raton, 2013).

[15] F. Wang and O. Korotkova, Circularly symmetric cusped random beams in free space and atmospheric turbulence, Opt. Express 25, 5057 (2017).

[16] J. Tervo and J. Turunen, Angular spectrum representation of partially coherent electromagnetic fields, Opt. Commun. 209, 7 (2002).

[17] R. Carminati and J.-J. Greffet, Near-Field Effects in Spatial Coherence of Thermal Sources, Phys. Rev. Lett. 82, 1660 (1999).

[18] A. V. Shchegrov, K. Joulain, R. Carminati, and J.-J. Greffet, Near-Field Spectral Effects due to Electromagnetic Surface Excitations, Phys. Rev. Lett. 85, 1548 (2000).

[19] M. Batarseh, S. Sukhov, Z. Shen, H. Gemar, R. Rezvani, and A. Dogariu, Passive sensing around the corner using spatial coherence, Nat. Commun. 9, 3629 (2018).

[20] W. Carter and E. Wolf, Coherence properties of lambertian and non-lambertian sources, J. Opt. Soc. Am. 65, 1067 (1975).

[21] M. Nyman, A. Shevchenko, I. Shavrin, Y. Ando, K. Lindfors, and M. Kaivola, Large-area enhancement of far-field fluorescence intensity using planar nanostructures, APL Photonics 4, 076101 (2019).

[22] R. J. Potton, Reciprocity in optics, Rep. Prog. Phys. 67, 717 (2004).

[23] S. C. Hill, G. Videen, and J. D. Pendleton, Reciprocity method for obtaining the far fields generated by a source inside or near a microparticle, J. Opt. Soc. Am. B 14, 2522 (1997). 
[24] E. Dieudonné, N. Malléjac, and S. Enoch, Scattering by complex inhomogeneous objects: a first-order reciprocity method, Opt. Express 22, 16558 (2014).

[25] M. Nyman, A. Shevchenko, and M. Kaivola, Fluorescence enhancement and nonreciprocal transmission of light waves by nanomaterial interfaces, Phys. Rev. A 96, 053828 (2017).
[26] B. Saleh and M. Teich, Fundamentals of Photonics (Wiley, Hoboken, 2007).

[27] L. Novotny and B. Hecht, Principles of Nano-Optics (Cambridge University Press, New York, 2012).

[28] E. Marchand and E. Wolf, Angular correlation and the far-zone behavior of partially coherent fields, J. Opt. Soc. Am. 62, 379 (1972). 